\title{
2D cine vs. 3D self-navigated free-breathing high-resolution whole heart cardiovascular magnetic resonance for aortic root measurements in congenital heart disease
}

\author{
Clément Nussbaumer ${ }^{1}$, Judith Bouchardy ${ }^{2}$, Coralie Blanche ${ }^{1}$, Davide Piccini ${ }^{3,4}$, Anna-Giulia Pavon',
} Pierre Monney ${ }^{1}$, Matthias Stuber ${ }^{3}$, Jürg Schwitter ${ }^{1}$ and Tobias Rutz ${ }^{1,2^{*}}$ (i)

\begin{abstract}
Background: Cardiovascular magnetic resonance (CMR) is considered the method of choice for evaluation of aortic root dilatation in congenital heart disease. Usually, a cross-sectional 2D cine stack is acquired perpendicular to the vessel's axis. However, this method requires a considerable patient collaboration and precise planning of image planes. The present study compares a recently introduced 3D self-navigated free-breathing high-resolution whole heart CMR sequence (3D self nav) allowing a multiplanar retrospective reconstruction of the aortic root as an alternative to the 2D cine technique for determination of aortic root diameters.

Methods: A total of 6 cusp-commissure (CuCo) and cusp-cusp (CuCu) enddiastolic diameters were measured by two observers on 2D cine and 3D self nav cross-sectional planes of the aortic root acquired on a 1.5 T CMR scanner. Asymmetry of the aortic root was evaluated by the ratio of the minimal to the maximum 3D self nav CuCu diameter. CuCu diameters were compared to standard transthoracic echocardiographic (TTE) aortic root diameters.

Results: Sixty-five exams in 58 patients (32 \pm 15 years) were included. Typically, 2D cine and 3D self nav spatial resolution was $1.1-1.5^{2} \times 4.5-7 \mathrm{~mm}$ and $0.9-1.15^{3} \mathrm{~mm}$, respectively. $3 \mathrm{D}$ self nav yielded larger maximum diameters than $2 \mathrm{D}$ cine: CuCo $37.2 \pm 6.4$ vs. $36.2 \pm 7.0 \mathrm{~mm}(p=0.006)$, CuCu $39.7 \pm 6.3$ vs. $38.5 \pm 6.5 \mathrm{~mm}$ ( $p<0.001)$. CuCu diameters were significantly larger $(2.3-3.9 \mathrm{~mm}, \mathrm{p}<0.001)$ than CuCo and TTE diameters on both 2D cine and 3D self nav. Intra- and interobserver variabilities were excellent for both techniques with bias of -0.5 to $1.0 \mathrm{~mm}$. Intra-observer variability of the more experienced observer was better for $3 \mathrm{D}$ self nav (F-test $\mathrm{p}<0.05$ ). Aortic root asymmetry was more pronounced in patients with bicuspid aortic valve (BAV: 0.73 (interquartile (IQ) $0.69 ; 0.78)$ vs. 0.93 (IQ 0.9; 0.96), $p<0.001$ ), which was associated to a larger difference of maximum CuCu to TTE diameters: $5.5 \pm 3.3$ vs. $3.3 \pm 3.8 \mathrm{~mm}, p=0.033$.

Conclusion: Both, the 3D self nav and 2D cine CMR techniques allow reliable determination of aortic root diameters. However, we propose to privilege the 3D self nav technique and measurement of CuCu diameters to avoid underestimation of the maximum diameter, particularly in patients with asymmetric aortic roots and/or BAV.
\end{abstract}

Keywords: Aortic root dilatation, 3D self-navigation whole heart, Congenital heart disease, Bicuspid aortic valve

\footnotetext{
*Correspondence: tobias.rutz@chuv.ch

${ }^{1}$ Service of Cardiology, Centre de Resonance Magnétique Cardiaque,

Lausanne University Hospital and University of Lausanne, Lausanne, Switzerland

Full list of author information is available at the end of the article
}

\section{Background}

Dilatation of the ascending aorta is frequently encountered in congenital heart disease (CHD) like bicuspid aortic valve (BAV) and hereditary aortopathies such as 
Marfan, Loeys-Dietz or Turner syndrome [1-4]. Regular imaging of the aorta is essential in these patients to time prophylactic surgical intervention for prevention of dramatic consequences such as aortic dissection [5-8]. To correctly evaluate the vessel diameters perpendicular to the vessel axis, three-dimensional (3D) imaging techniques like cardiovascular magnetic resonance (CMR) or cardiac computed tomography (CCT) are used. Bright and dark blood CMR techniques have been introduced for assessment of the aorta. Black blood T1-and T2-weighted sequences allow assessment of the aorta and its wall without the administration of a contrast agent $[9,10]$. Typical bright blood sequences are the contrast-enhanced angiography, providing a 3D data set of the aorta, and the fast gradient echo (GRE) or the balanced steady-state free precession (bSSFP) sequences, the two latter sequences not requiring contrast administration. As the aortic root presents an important pulsatility and translational motion of up to $2 \mathrm{~cm}$ during the cardiac cycle, electrocardiogram (ECG)-gated imaging techniques are required to avoid blurring of the vessel wall $[11,12]$. There is, however, no uniform method to measure aortic root diameters and practice variation exists with regard to determination of leading to leading (L-L), inner to inner (I-I) or outer to outer $(\mathrm{O}-\mathrm{O})$ edge diameters as well as measurements of cusp to commissure $(\mathrm{CuCo})$ or cusp to cusp $(\mathrm{CuCu})$ diameters $[11,13$, 14]. When using CMR, a stack of $2 \mathrm{D}$ cines is prescribed in doubly-oblique cross-sectional orientation on two orthogonal long axis cines of the left ventricular (LV) outflow tract (LVOT) and perpendicular to the axis of the aortic root (Fig. 1a) [14]. This technique requires, however, a careful planning of the 2D cine stack to limit variability of measurements. In addition, repetitive breath-holds during the acquisition of the 2D cines are necessary, which can be difficult for populations with suboptimal compliance such as patients with trisomy 21 , intellectual deficit or dyspnea. An alternative is the use of a 3D self-navigated free-breathing high-resolution whole heart CMR sequence (3D self nav) with either end-systolic or diastolic gating [15]. This technique, which has been introduced by Piccini et al. in 2012, uses a radial readout extracting the respiratory motion data directly at the level of the heart and from the k-space data [16-18]. The use of a 3D radial readout allows achieving a high isovolumetric spatial resolution as well as acquiring the self-navigating readout at each heart beat while maintaining uniformity in the sampling. The trajectory has a kooshball like arrangement of the readouts, in which every single $\mathrm{k}$-space line crosses the center of k-space. The geometrical distribution of the readouts follows a spiral phyllotaxis patterns as described previously [18]. The advantage of the latter method is a free-breathing image acquisition without the need for meticulous scan plane adaptations and respiratory navigator placement, thus leading to a relatively simple exam with $100 \%$ scan efficiency and a predictable image acquisition duration, all of which rendering the exam very comfortable for the patient $[17,19]$. The acquired high-resolution 3D whole heart volume allows a flexible retrospective multiplanar reconstruction (MPR) of the image plane perpendicular to the vessel's axis for determination of the aortic root diameters.

The aim of the present study was therefore to compare the precision and the reliability of both the $2 \mathrm{D}$ cine and 3D self nav methods in determining aortic root diameters in CHD patients with different aortic pathologies.

\section{Methods}

This is a retrospective study on CMR exams performed for aortic root evaluation at a single institution. The study was approved by the local ethics committee with waiver of informed consent.

\section{Population}

Patients undergoing CMR for evaluation of the aortic root between 2014 and 2019 by both types of CMR sequences, 2D cine and 3D self nav, were included. Both sequences were part of the routine imaging protocol in these patients. Patients with data of only one of the two above-mentioned CMR sequences were excluded.

\section{CMR Imaging}

Patients were scanned with two $1.5 \mathrm{~T}$ clinical CMR scanners (MAGNETOM Aera and Sola, Siemens Healthineers, Erlangen, Germany) with a 30-channel phased-array coil. The imaging protocol was selected according to the specific malformation. [14, 20] Contrast medium was used in all patients (Gadobutrol, Gadovist ${ }^{\circledR}$, Bayer Healthcare, Berlin, Germany, dose: $0.2 \mathrm{mmol} / \mathrm{kg}$ ). No heart rate lowering medication was used.

\section{D cine CMR Imaging}

To acquire the 2D cine stack in doubly-oblique, crosssectional orientation to the aortic root axis, first a standard 3-chamber long axis cine of the was obtained [20]. Second, an LVOT cine was prescribed orthogonal to the 3 -chamber view. Third, a stack of eight to ten $2 \mathrm{D}$ cines without gap of the aortic root was planned perpendicular to the aortic root axis on both, the 3 chamber and LVOT cines (Fig. 1a). 2D cines were acquired using either a fast gradient echo (GRE) or a bSSFP sequence. Use of GRE was chosen by the physician in charge of the CMR exam in case of relevant image artifacts on bSSFP. Typical imaging parameters for GRE and bSSFP sequences were matrix 256/146, slice thickness $4.5-7 \mathrm{~mm}$, in-plane 

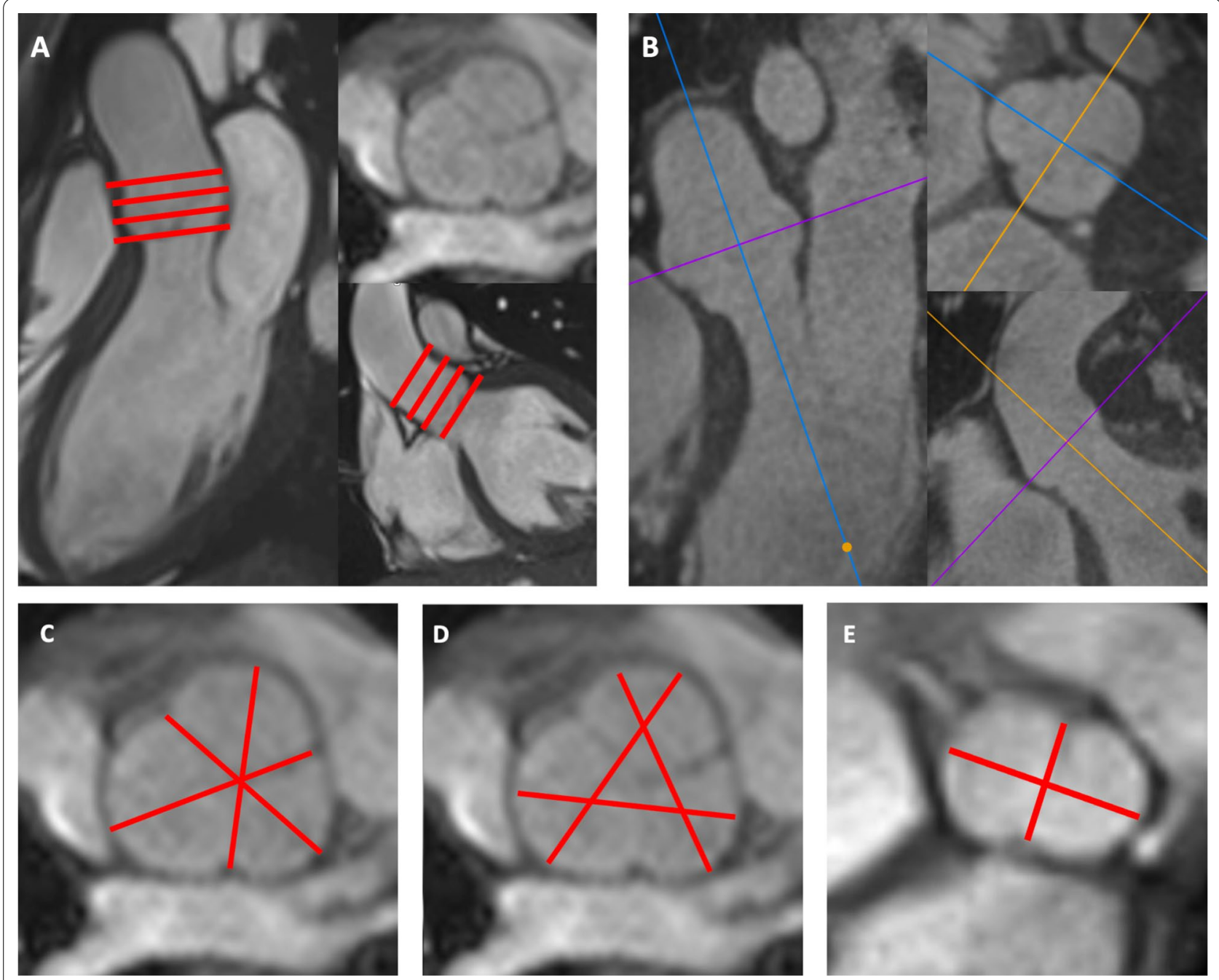

Fig. 1 The doubly-oblique cross-sectional stack of 2D cines was planned on two orthogonal cines of the left ventricular outflow tract (LVOT) (a). For 3D self nav, a multiplanar reconstruction of the aortic root plane was performed (b). Cusp to the opposite commissure (CuCo) (c) and cusp to cusp (CuCu, d) inner to inner edge diameters were determined. For bicuspid aortic valve (BAV) patients in whom identification of three cusps was not possible, the maximum diameter and a second, orthogonal diameter were obtained (e)

resolution 1.1-1.5 $\mathrm{mm}$, temporal resolution $<50 \mathrm{~ms}$, duration of breath-holding about 8 to $12 \mathrm{~s}$. Specific parameters for GRE were TR/TE $51 / 3.5 \mathrm{~ms}$, flip angle $15^{\circ}$; for bSSFP TR/TE $45 / 1.3 \mathrm{~ms}$, flip angle $59^{\circ}$.

\section{D self-navigated free-breathing high-resolution whole heart CMR}

The prototype 3D self nav sequence was acquired as previously described for CHD patients [15]. The 3D self nav CMR sequence was started after injection of the contrast agent for the 3D contrast-enhanced angiography. The imaging parameters were as follows: TR/TE $3.1 / 1.56 \mathrm{~ms}$, field-of-view $200-220^{3} \mathrm{~mm}$, matrix $192^{3}$, receiver bandwidth $900 \mathrm{~Hz} /$ pixel, isotropic spatial resolution (both acquired and reconstructed) $0.9-1.15 \mathrm{~mm}$, about $12^{\prime} 000$ radial readouts, acquisition duration $8-10 \mathrm{~min}$. The trigger delay was visually identified at the most quiescent mid- to end-diastolic period on a cine 4 chamber view.

\section{CMR image analyses}

Two observers, one with $<1$-year experience in CMR (NC, observer 1), and one experienced reader (TR, observer 2, EuroCMR level III certified in general and congenital CMR), performed the measurements of aortic root diameters. They were blinded to diagnosis and previous medical surgery of patients. Prior to the aortic diameter measurements, observer 1 trained for about 
$20 \mathrm{~h}$ to analyze the CMR images under the supervision of observer 2 .

\section{CMR image quality}

Image quality was assessed for each CMR acquisition technique according to a five-point scale as previously described [15]: Grade 5 corresponds to an excellent and grade 4 to a good diagnostic quality with mild blurring, whereas grade 3 indicates diagnostic quality, despite moderate blurring of cardiac and vascular structures. Grade 2 indicates marked blurring of the structures, preventing a complete anatomical diagnosis. In grade 1 , a dataset was considered non-diagnostic.

\section{CMR aortic root diameter measurement}

Aortic root measurements were performed on Syngo. $\mathrm{Via}^{\mathrm{TM}}$ (Siemens Healthineers). For measurement on 2D cines, each observer chose the adequate slice of the 2D cine stack representing the maximal aortic diameter at end-diastole (Fig. 1a). For 3D self nav measurements, each observer performed a MPR of the image plane representing the largest aortic root diameters (Fig. 1b). For both, 2D cine and 3D self nav, a total of six I-I diameters were measured: three $\mathrm{CuCo}$ and three $\mathrm{CuCu}$ diameters (Fig. 1c, d). Diameters were classified as minimal, mid and maximum diameter according to their measured length. For 18 (28\%) patients with apparent "real" BAV, identification of three cusps was not possible on $2 \mathrm{D}$ cine and $3 \mathrm{D}$ self nav. For these patients, the maximal diameter and a second, orthogonal diameter were obtained as previously described (Fig. 1e). [21] Of note, for 3 patients, identification of three cusps was only possible on one imaging technique (observer 1: two patients on $3 \mathrm{D}$ self nav, one patient on $2 \mathrm{D}$ cine; observer 2: 1 patient on 3D self nav). Observer 1 performed both, $\mathrm{CuCo}$ and $\mathrm{CuCu}$ measurements twice for all patients. Observer 2 performed the same measurements twice for 20 randomly chosen patients allowing to assess the inter-observer variability and the intraobserver variabilities for both observers.

An asymmetry index of the aortic root was calculated by obtaining the ratio of the minimal to the maximum 3D self nav $\mathrm{CuCu}$ diameter.

\section{Transthoracic echocardiographic (TTE) aortic root diameter measurement}

Results of 2D aortic root measurements were obtained from TTE exams performed within 6 months of the CMR. In our institution, a standard 2D parasternal long-axis view is obtained with a zoom on the aortic root. The end-diastolic frame is chosen, and the diameter of the aortic root measured using the L-L technique as proposed by the guidelines of the American Society of Echocardiography (ASE) and European Association of Cardiovascular Imaging (EACVI). [11].

\section{Statistical analysis}

Normality was tested with the Shapiro-Wilk test. Parameters are indicated as mean \pm standard deviation (SD) or as number and percentage, where appropriate. Wilcoxon signed-rank test was used to assess differences in the scoring of image quality. Bland-Altman analyses were performed to compare 2D cine and 3D self nav diameters. [22] Bland-Altman and intraclass correlation coefficient (ICC) analyses were performed to evaluate intra- and inter-observer variabilities of diameter measurements of 2D cine and 3D self nav. Paired t-tests or Wilcoxon signed-rank tests, where appropriate, were performed for comparison of continuous parameters and F-test for comparison of variances. Pearson's correlation was used to determine the correlation between parameters.

Influence of baseline parameters (image quality, use of GRE, BAV, prior aortic valve surgery, presence of a genetic syndrome, $\mathrm{AI}$ ) on comparisons of $2 \mathrm{D}$ cine and 3D self nav diameters, intra- and inter-observer variabilities were determined by t-tests or Pearson's correlation. Statistical analysis was performed using SPSS (version 26.0, Statistical Package for the Social Sciences, International Business Machines, Inc., Armonk, New York, USA), Excel (version 16.35, Microsoft, Redmond, Washington, USA) and Graphpad Prism (version 5, Graphpad Software, San Diego, California, USA).

\section{Results}

\section{Study population}

Sixty-five CMR exams from 58 patients were included (reasons for two CMR exams in 7 patients are indicated in Additional file 1). Baseline characteristics of the population are shown in Table 1. Patient population consisted of four groups: non-operated aortic pathologies (BAV 28\%, coarctation of the aorta 5\%, subvalvular aortic membrane $3 \%$ ), prior aortic valve or root surgery (Ross procedure $22 \%$, Tirone David Procedure $8 \%$, commissurotomy $8 \%$, Hemashield Graft 3\%, Bentall procedure $2 \%$ ), syndromic patients (Marfan syndrome 14\%, Turner syndrome 3\%, Ehlers-Danlos' syndrome 2\%) and patients undergoing CMR for work-up of the ascending aorta (5\%). GRE was used in 19 (29\%) of exams. 
Table 1 Patients' characteristics

\begin{tabular}{|c|c|c|}
\hline & $\begin{array}{l}\text { Total } \\
\text { cohort }\end{array}$ & \\
\hline & $N=65$ & \\
\hline Patient characteristics & & \\
\hline Age & Years & $32.3 \pm 14.6$ \\
\hline Male gender & N (\%) & $42(64.6 \%)$ \\
\hline Height & $\mathrm{cm}$ & $174.7 \pm 11.5$ \\
\hline Weight & $\mathrm{kg}$ & $69.5 \pm 16.8$ \\
\hline BSA & $m^{2}$ & $1.83 \pm 0.24$ \\
\hline Arterial hypertension & $\mathrm{N}(\%)$ & $13(20.0 \%)$ \\
\hline Patient diagnosis & & \\
\hline Prior aortic root surgery & $\mathrm{N}(\%)$ & $27(41.5 \%)$ \\
\hline Time since surgery & Years & $12.3 \pm 7.9$ \\
\hline Non-operated aortic pathologies & N (\%) & $23(35.4 \%)$ \\
\hline Genetic syndrome & N (\%) & $12(18.5 \%)$ \\
\hline Ascending aorta assessment & N (\%) & $3(4.6 \%)$ \\
\hline Aortic valve characteristics & & \\
\hline Bicuspid aortic valve & $\mathrm{N}(\%)$ & $24(36.9 \%)$ \\
\hline CMR aortic regurgitant fraction & $\%$ & $9.4 \pm 12.4$ \\
\hline Aortic regurgitation by TTE & $\mathrm{N}(\%)$ & $45(69.2 \%)$ \\
\hline Grade 1 & & $26(40.0 \%)$ \\
\hline Grade 2 & & $14(21.5 \%)$ \\
\hline Grade 3 & & $5(7.7 \%)$ \\
\hline Aortic stenosis & N (\%) & $12(18.5 \%)$ \\
\hline Maximal aortic gradient & $\mathrm{mmHg}$ & $12.3 \pm 10.7$ \\
\hline Mean aortic gradient & $\mathrm{mmHg}$ & $6.2 \pm 5.5$ \\
\hline Maximal aortic velocity & $\mathrm{cm} / \mathrm{s}$ & $158.0 \pm 66.3$ \\
\hline Aortic diameters & & \\
\hline Aortic root diameter on TTE & $\mathrm{mm}$ & $36.0 \pm 7.9$ \\
\hline$C M R^{a}$ & & \\
\hline $\begin{array}{l}\text { Ascending aorta level of right pulmonary } \\
\text { artery }\end{array}$ & $\mathrm{mm} / \mathrm{m}^{2}$ & $17.5 \pm 4.5$ \\
\hline Aortic arch & $\mathrm{mm} / \mathrm{m}^{2}$ & $12.5 \pm 2.4$ \\
\hline Descending aorta level of diaphragm & $\mathrm{mm} / \mathrm{m}^{2}$ & \pm 1.9 \\
\hline
\end{tabular}

BSA body surface area, TTE transthoracic echocardiography

${ }^{\text {a }}$ Measured on either a standard contrast-enhanced 3D angiography or 3D self nav

\section{CMR image quality}

The image quality did not differ between 2D cine and 3D self nav ( $\mathrm{p}=0.857$, Fig. 2 ) nor between both observers: $p=0.705$ for $2 \mathrm{D}$ cine and $p=0.221$ in $3 \mathrm{D}$ self nav.

\section{Aortic root measurement}

Although Pearson's correlation analyses showed a strong correlation of diameters obtained by both, 2D cine and 3D self nav $(r=0.94-0.97)$, aortic root diameters were systematically larger on $3 \mathrm{D}$ self nav compared to 2D cine (range $=0.8-1.3 \mathrm{~mm}$ for observer $1, \mathrm{p}<0.01$, Table 2 and Table S1). Figure 3a, b show the Bland-Altman plots for the comparison of the

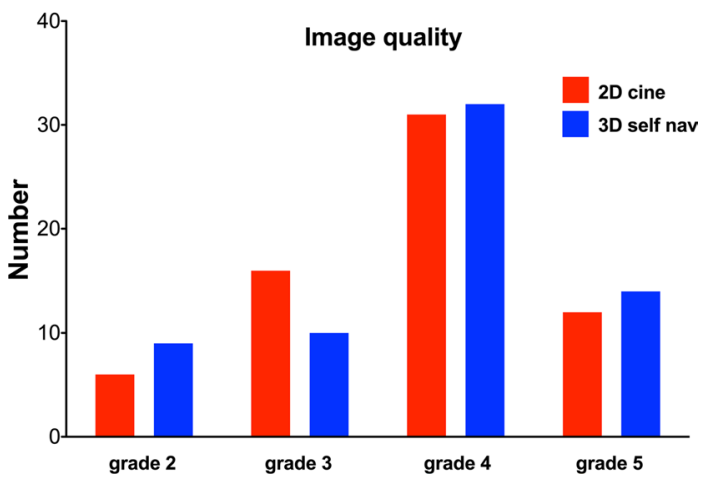

Fig. 2 Comparison of image quality of 2D cine (red bars) and 3D self nav (blue bars). There were no differences of image quality neither between techniques nor between observers $(p>0.05)$

maximum $\mathrm{CuCo}$ and $\mathrm{CuCu}$ diameters measured on 2D cine vs. 3D self nav.

$\mathrm{CuCu}$ diameters were systematically 2.3 to $2.9 \mathrm{~mm}$ larger than the $\mathrm{CuCo}$ diameters on both $2 \mathrm{D}$ cine and 3D self nav $(p<0.001)$. Detailed results are presented in Table 3 and Fig. 3c, d. Results on comparison of minimal and mid diameters between 2D cine and 3D self nav as well as $\mathrm{CuCu}$ and $\mathrm{CuCo}$ are provided in Additional file 1: Tables S1, S2).

Intra-observer variabilities were excellent for both techniques with a mean bias of $\leq 0.6 \mathrm{~mm}$ for both observers and both, the 2D cine and 3D self nav techniques (Table 4 and Table S3, Additional file 1). ICC values were excellent for both observers. For observer 1, variances were smaller for determination of maximum $\mathrm{CuCo} 2 \mathrm{D}$ cine vs. 3D self nav diameters. For observer 2, variances were smaller on 3D self nav for both, maximum $\mathrm{CuCo}$ and $\mathrm{CuCu}$ diameters ( $\mathrm{F}$ test, $p<0.05$ ). Bland-Altman plots of intra-observer variabilities of both observers for maximum $\mathrm{CuCo}$ and $\mathrm{CuCu}$ diameters are provided in Additional file 1.

The inter-observer variability was excellent with mean differences ranging from $0.1 \mathrm{~mm}$ to $0.8 \mathrm{~mm}$ for $2 \mathrm{D}$ cine and $-0.3 \mathrm{~mm}$ to $1.0 \mathrm{~mm}$ for 3D self nav, respectively (for maximum diameters see Table 5 and Additional file 1: Figure S3, for minimal and mid diameters see Additional file 1: Table S4). ICC values were excellent, and bias and variances did not differ significantly ( $p>0.05)$.

TTE aortic root diameters were available for all but two patients. While maximum 2D cine and maximum 3D self nav CuCo diameters did not differ significantly to maximum TTE aortic root diameters, maximum $\mathrm{CuCu}$ diameters were significantly larger on both, 2D cine and $3 \mathrm{D}$ self nav measurements with larger biases for diameters measured on 3D self nav (Table 6). The differences between TTE and maximum $\mathrm{CuCu}$ diameters were larger 
Table 2 Comparison of maximum 2D cine CMR vs 3D self nav CMR aortic root diameters

\begin{tabular}{|c|c|c|c|c|}
\hline \multirow[t]{2}{*}{ 2D cine vs. 3D self nav } & \multicolumn{2}{|l|}{ Observer 1} & \multicolumn{2}{|l|}{ Observer 2} \\
\hline & CuCo max & CuCu max & CuCo max & CuCu max \\
\hline Mean diameter 2D cine (mm) & 36.2 & 38.5 & 35.8 & 38.2 \\
\hline Mean diameter 3D self nav $(\mathrm{mm})$ & 37.2 & 39.7 & 37.3 & 39.5 \\
\hline Mean difference (mm) & -1.0 & -1.2 & -1.6 & -1.3 \\
\hline 95\% Limits of agreement (mm) & -5.5 to 3.5 & -4.7 to 2.3 & -4.7 to 1.4 & -5.0 to 2.4 \\
\hline Standard deviation (mm) & 2.3 & 1.8 & 1.5 & 1.9 \\
\hline Variance $\left(\mathrm{mm}^{2}\right)$ & 5.2 & 3.2 & 2.0 & 3.6 \\
\hline Pearson's correlation $(r)$ & 0.945 & 0.951 & 0.985 & 0.964 \\
\hline$P$ value (t-test) & 0.006 & $<0.001$ & 0.001 & 0.009 \\
\hline
\end{tabular}

3D self nav 3D self-navigated high-resolution free-breathing whole heart, CuCo cusp to commissure, CuCu cusp to cusp, max maximum

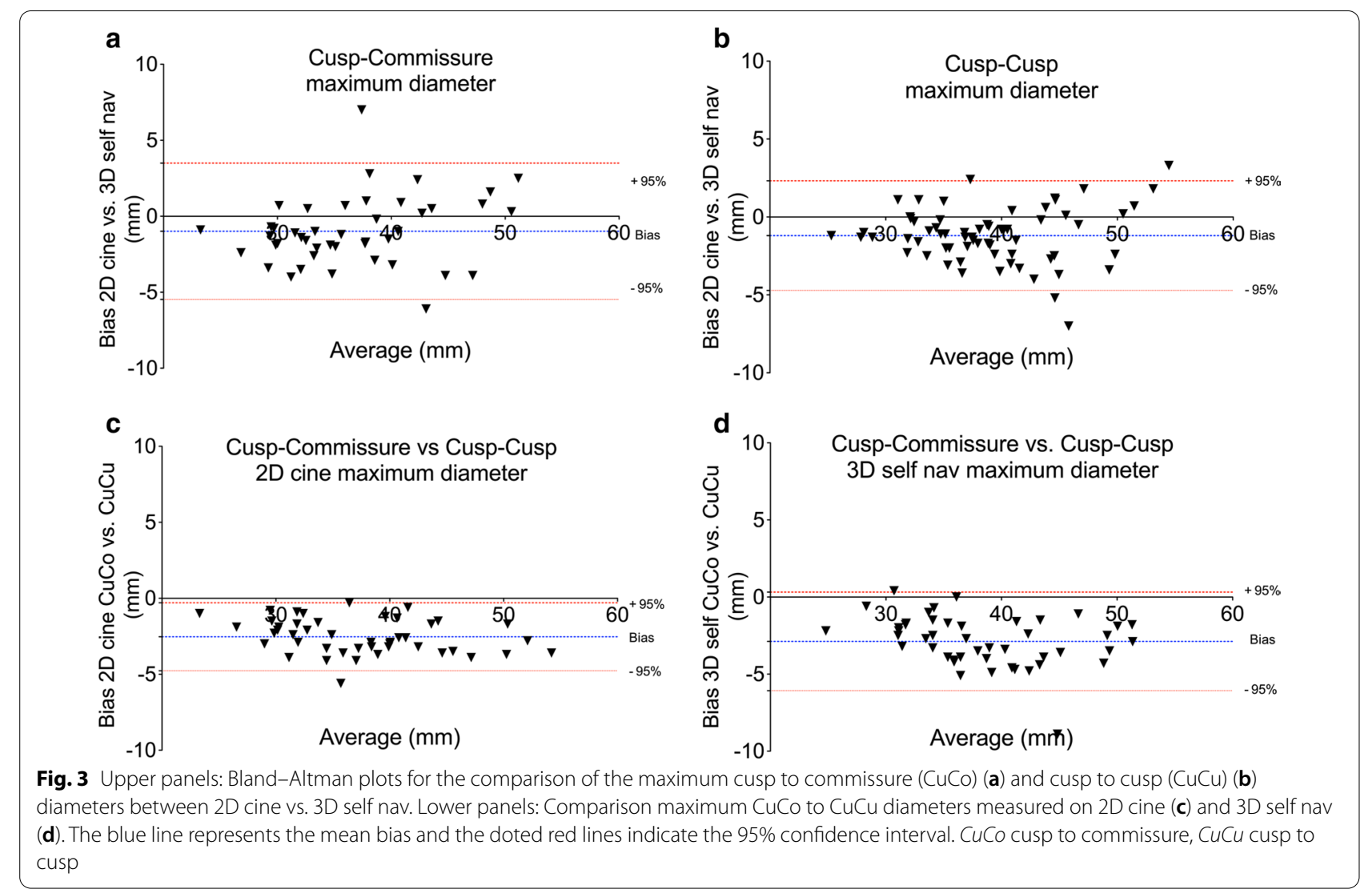

in BAV patients $(5.5 \pm 3.3$ vs. $3.3 \pm 3.8 \mathrm{~mm}, \mathrm{p}=0.033)$. Of note, the decision on identification of three cusps did not match in 3 cases for observer 1 and in one case for observer 2. As patients without three identified cusps were allocated to the $\mathrm{CuCu}$ measurements and for two patients no TTE diameters were available, resulting TTE diameters in Table 6 differ slightly between analyses when using paired t-tests.
Significant aortic root asymmetry was found in 15 (23\%) of included patients when using an asymmetry index cut-off of $<0.75$. AI was significantly smaller in BAV than in non-BAV patients: 0.73 (interquartile (IQ) 0.69; 0.78 ) vs. 0.93 (IQ 0.9; 0.96), $\mathrm{p}<0.001$ ). A more severe asymmetry was associated with a larger discrepancy of TTE aortic root diameters to maximum $\mathrm{CuCu} 3 \mathrm{D}$ self nav diameter: $\mathrm{AI}<0.75(\mathrm{~N}=15)$ vs. $\geq 0.75(\mathrm{~N}=48)=5.6 \pm 3.4$ 
Table 3 Comparison of maximum Cusp to Commissure vs. Cusp to Cusp diameters

\begin{tabular}{lll}
\hline & 2D cine & $\begin{array}{l}\text { 3D self } \\
\text { nav }\end{array}$ \\
\hline Mean diameter CuCo (mm \pm SD) & $36.2 \pm 6.9$ & $37.0 \pm 6.3$ \\
Mean diameter CuCu (mm \pm SD) & $38.7 \pm 7.2$ & $39.9 \pm 6.8$ \\
Bias (mm) & -2.5 & -2.9 \\
Standard deviation (mm) & 1.1 & 1.6 \\
$95 \%$ Limits of agreement $(\mathrm{mm})$ & $-4.8 ;-0.3$ & $-6.1 ; 0.3$ \\
Variance $\left(\mathrm{mm}^{2}\right)$ & 1.3 & 2.6 \\
Pearson's correlation $(\mathrm{r})$ & 0.988 & 0.972 \\
P value (t-test) & $<0.001$ & $<0.001$ \\
\hline
\end{tabular}

CuCo cusp to commissure, CuCu cusp to cusp

vs. $3.4 \pm 3.8 \mathrm{~mm}, \mathrm{p}=0.047$. Of note, all but one patient with an asymmetry index $<0.75$ were BAV patients.

\section{Further supplementary material}

We evaluated parameters influencing the precision of $2 \mathrm{D}$ cine and 3D self nav measurements. There was no significant impact of image quality or the use of GRE on comparison of 2D cine vs. 3D self nav measurements nor on intra- or inter-observer variabilities $(p>0.05)$. Aortic root surgery, asymmetry index, maximum aortic root diameter, presence of BAV and genetic syndrome influenced the bias and reliability of some diameter determinations, for details please see tables S5-7 in Additional file 1. Of note that aortic root surgery did not influence the image quality.

\section{Discussion}

This study systematically compares two CMR sequences for aortic root evaluation in patients with CHD. We provide four important findings to physicians performing CMR in this patient population:

$12 \mathrm{D}$ cine and 3D self nav are both reliable methods for aortic root measurements with only small differences in precision.

2 3D self nav provides significantly larger diameters than $2 \mathrm{D}$ cine.

Table 5 Interobserver variability

\begin{tabular}{lll}
\hline & CuCo max & CuCu max \\
\hline 2D cine & & \\
Mean difference (mm) & 0.5 & 0.6 \\
95\% Limits of agreement (mm) & -2.9 to 3.9 & -2.7 to 3.8 \\
Standard deviation (mm) & 1.7 & 1.6 \\
Variance (mm2) & 3.0 & 2.7 \\
ICC & 0.966 & 0.983 \\
3D self nav & & \\
Mean difference (mm) & -0.3 & 1.0 \\
95\% Limits of agreement (mm) & -6.4 to 5.8 & -2.6 to 4.5 \\
Standard deviation (mm) & 3.1 & 1.8 \\
Variance (mm2) & 9.7 & 3.2 \\
ICC & 0.950 & 0.978 \\
P value (t-test) & 0.484 & 0.495 \\
p-value (F test) & 0.139 & 0.910 \\
\hline Abbriations see Tabs 3 and 4 &
\end{tabular}

Abbreviations: see Tables 3 and 4

Table 4 Intra-observer variability

\begin{tabular}{|c|c|c|c|c|}
\hline & \multicolumn{2}{|l|}{ Observer 1} & \multicolumn{2}{|l|}{ Observer 2} \\
\hline & CuCo max & CuCu max & CuCo max & CuCu max \\
\hline \multicolumn{5}{|l|}{$2 D$ cine } \\
\hline Mean difference (mm) & -0.4 & -0.3 & -0.1 & -0.3 \\
\hline 95\% Limits of agreement $(\mathrm{mm})$ & -3.0 to 2.1 & -2.7 to 2.1 & -3.9 to 3.6 & -3.5 to 2.9 \\
\hline Standard deviation (mm) & 1.3 & 1.2 & 1.9 & 1.6 \\
\hline Variance $\left(\mathrm{mm}^{2}\right)$ & 1.7 & 1.5 & 3.6 & 2.6 \\
\hline ICC & 0.989 & 0.991 & 0.975 & 0.985 \\
\hline \multicolumn{5}{|l|}{ 3D selfnav } \\
\hline Mean difference $(\mathrm{mm})$ & -0.6 & -0.6 & -0.1 & -0.4 \\
\hline 95\% Limits of agreement $(\mathrm{mm})$ & -5.3 to 4.0 & -3.4 to 2.2 & -1.9 to 2.0 & -2.3 to 1.5 \\
\hline Standard deviation (mm) & 2.4 & 1.4 & 1.0 & 1.0 \\
\hline Variance $\left(\mathrm{mm}^{2}\right)$ & 5.6 & 2.0 & 1.0 & 1.0 \\
\hline ICC & 0.965 & 0.971 & 0.994 & 0.994 \\
\hline$P$ value & 0.884 & 0.268 & 0.712 & 0.993 \\
\hline$p$-value ( $F$ test) & 0.047 & 0.336 & 0.020 & 0.034 \\
\hline
\end{tabular}

ICC interclass correlation coefficient, others see Table 3 
Table 6 Comparison of aortic root diameters between echocardiography and cardiac magnetic resonance

\begin{tabular}{|c|c|c|c|c|c|}
\hline TTE vs. 2D cine & CuCo max & CuCu max & TTE vs. 3D self nav & CuCo max & CuCu max \\
\hline Observer 1 & & & Observer 1 & & \\
\hline Mean diameter TTE (mm) & 37.2 & 36.0 & Mean diameter TTE (mm) & 37.0 & 36.0 \\
\hline Mean diameter 2D cine (mm) & 36.6 & 38.4 & Mean diameter $3 \mathrm{D}$ self nav $(\mathrm{mm})$ & 37.4 & 39.9 \\
\hline Mean difference (mm) & -0.6 & 2.3 & Mean difference (mm) & 0.4 & 3.9 \\
\hline Limits of agreement (mm) & -7.4 to 6.2 & -4.4 to 9.1 & Limits of agreement (mm) & -8.0 to 8.7 & -3.5 to 11.3 \\
\hline Standard deviation (mm) & 3.5 & 3.4 & Standard deviation (mm) & 4.2 & 3.8 \\
\hline Variance $\left(\mathrm{mm}^{2}\right)$ & 12.1 & 11.8 & Variance $\left(\mathrm{mm}^{2}\right)$ & 18.0 & 14.3 \\
\hline Pearson's correlation ( $r$ ) & 0.972 & 0.903 & Pearson's correlation ( $r$ ) & 0.882 & 0.881 \\
\hline$P$ value (t-test) & 0.247 & $<0.001$ & $P$ value (t-test) & 0.564 & $<0.001$ \\
\hline Observer 2 & & & Observer 2 & & \\
\hline Mean diameter TTE (mm) & 36.9 & 36.6 & Mean diameter TTE (mm) & 36.6 & 36.6 \\
\hline Mean diameter 2D cine (mm) & 36.1 & 38.4 & Mean diameter $3 \mathrm{D}$ self nav $(\mathrm{mm})$ & 37.8 & 39.9 \\
\hline Mean difference (mm) & -0.8 & 1.8 & Mean difference (mm) & 1.1 & 3.4 \\
\hline Limits of agreement (mm) & -9.7 to 8.2 & -6.0 to 9.6 & Limits of agreement (mm) & -5.9 to 8.2 & -3.1 to 9.9 \\
\hline Standard deviation (mm) & 4.6 & 4.0 & Standard deviation (mm) & 3.6 & 3.3 \\
\hline Variance $\left(\mathrm{mm}^{2}\right)$ & 20.8 & 15.9 & Variance $\left(\mathrm{mm}^{2}\right)$ & 12.8 & 10.9 \\
\hline Pearson's correlation ( $r$ ) & 0.881 & 0.869 & Pearson's correlation ( $r$ ) & 0.932 & 0.914 \\
\hline$P$ value (t-test) & 0.532 & 0.065 & $P$ value (t-test) & 0.221 & $<0.001$ \\
\hline
\end{tabular}

Of note, the decision on identification of three cusps did not match in 3 cases for observer 1 and in one case for observer 2 . As patients without three identified cusps were allocated to the CuCu measurements and for two patients no TTE diameters were available, resulting TTE diameters in Table 6 differ slightly between analyses when using paired t-tests

TTE transthoracic echocardiography, other see Tables 3 and 4

$3 \mathrm{CuCu}$ diameters are significantly larger than $\mathrm{CuCo}$ diameters on both techniques.

4. TTE diameters are systemically smaller than the maximum $\mathrm{CuCu}$ diameters. The difference is highly influenced by the aortic root asymmetry and the presence of BAV.

\section{Precision of 2D cine and 3D self nav}

Although aortic root dilatation has an important impact on mortality and morbidity in CHD patients, guidelines on image acquisition, choice and measurement of aortic root diameters are relatively vague $[6,8,23]$. Due to the important pulsatility and throughplane motion of the aortic root, ECG-gated imaging techniques are required [12]. Studies on precision of different CMR aortic root evaluation techniques are, however, surprisingly scarce [24-27]. Burman et al. determined normal values for aortic root diameters in healthy individuals using sagittal, coronal and perpendicular cross-sectional 2D cines of the aortic root suggesting the use of cross-sectional 2D cine and measurement of $\mathrm{CuCo}$ diameters [25]. Unfortunately, this very elegant study did not provide information on the precision of the applied imaging and measurement methods. Several studies compared nontriggered angiography with 2D cine or 3D bSSFP whole heart CMR, the latter usually ECG triggered and using a respiratory navigator $[24,26,28]$. For the $2 \mathrm{D}$ cine technique, a relatively large range of biases of 0.2 to $4 \mathrm{~mm}$ was reported for inter- and intra-observer variabilities [21, 26, 27, 29]. Further studies compared 3D bSSFP whole heart sequences using respiratory navigators to either 3D ECG gated angiography or TTE $[24,30,31]$. Veldhoen et al. did not observe differences for the precision of aortic root measurement by CMR 3D angiography and ECG gated 2D bSSFP, which is surprising but probably explained by the fact that only Marfan patients after aortic root replacement were included. One can speculate that the pulsatility and throughplane motion of the aortic root is diminished after surgery explaining why a non-triggered sequence like an 3D angiography performed relatively well [24]. Potthast showed that 3D bSSFP whole heart performed best for vessel diameter determination when compared to 3D contrast-enhanced CMR angiography, 2D T2 black blood, and 2D cine bSSFP [10].

All the above-cited studies used 3D bSSFP sequences with pencil beam respiratory navigators for respiratory motion correction. As an alternative, 3D self-navigation techniques have been developed operating without a reference position and extracting the respiratory motion at the heart and from the $\mathrm{k}$-space data $[17,19,32]$. The $3 \mathrm{D}$ self nav sequence used in this study offers a high spatial resolution with an isovolumetric voxel size of about $1.1 \mathrm{~mm}^{3}$ in contrast to most studies using $2 \mathrm{D}$ or $3 \mathrm{D}$ 
bSSFP sequences and its acquisition duration is predictable $[10,24,29,33]$. The potential of the 3D self nav has been shown for imaging of coronary arteries, evaluation of anatomy in CHD and detection of myocardial scar $[15,17,34]$. In the present study, the image quality was comparable between both sequences (Fig. 2) and, with respect to the 3D self nav sequence, similar to what our group has previously published [15]. Intra- and interobserver variabilities were excellent as expressed by a small bias of $\leq 1 \mathrm{~mm}$ and small limits of agreements comparable to previously published reports on reproducibility of $2 \mathrm{D}$ cine and 3D whole heart techniques $[3,15$, $24,26]$. We observed a better reproducibility for the 3D self nav method for the maximum diameter determination for the experienced observer. This finding is probably explained by the fact that performing MPRs on 3D self nav in complex anatomy is challenging for less experienced observers in contrast to "simply" choosing the $2 \mathrm{D}$ cine slice representing the largest diameter. Of note that all CMR exams were performed by physicians experienced in CMR (EuroCMR level III certified), assuring the correct orientation of 2D cines, which may have contributed to the high agreement observed between 2D cine and 3D self nav.

Diameters were systematically about $1 \mathrm{~mm}$ smaller on 2D cines compared to 3D self nav. Two factors probably explain this observation. First, the lower spatial resolution (both, inplane resolution and slice thickness) of 2D cines compared to the 3D self nav, although the 2D cine spatial resolution was similar to previous studies [25]. The resulting blurring of the aortic wall due to the partial volume effect results in an underestimation of the maximum diameters. Second, the timing of the 3D self nav to the most-quiescent diastolic period i.e. the mid to the end-diastolic phase as well as the higher temporal resolution of the 2D cine, might also contribute to the slightly larger diameters obtained by the 3D self nav sequence.

The precision of $2 \mathrm{D}$ cine and $3 \mathrm{D}$ self nav was influenced neither by the image quality nor using GRE for the $2 \mathrm{D}$ cine sequence. Aortic root surgery and $\mathrm{AI}$ were associated with a better agreement of $2 \mathrm{D}$ cine and $3 \mathrm{D}$ self nav diameters for observer 1 but not for observer 2 . This might be explained by the fact that the aortic root presents less pulsatility and throughplane motion after surgery reducing the variability between both methods.

Identification of the aortic root diameter appears to be more challenging in asymmetric roots, explaining the influence of asymmetry index, the maximum aortic root diameter, BAV and genetic syndrome on some, however, only few measurements.

\section{Which aortic root diameter should be measured by which method?}

Most long-standing recommendations on aortic disease are historically based on TTE L-L measurements. Several recent international and national guidelines tried to uniform the recommendations on aortic root evaluation $[5,11,13,14,35,36]$. The 2015 ASE/EACVI guidelines for chamber quantification suggest for CMR and $\mathrm{CT}$ both $\mathrm{CuCo}$ and $\mathrm{CuCu}$ measurements [11]. However, this same document as well as the 2014 European Society of Cardiology guidelines on aortic disease elucidate that it was not possible to obtain uniformity between different imaging modalities and currently, recommendations vary suggesting $\mathrm{CuCu}, \mathrm{CuCo}$ as well as I-I, $\mathrm{L}-\mathrm{L}$ and $\mathrm{O}-\mathrm{O}$ diameters and different image planes and sequences $[5,11]$. A further problem is that in studies identifying cut-offs for surgical intervention in e.g. Marfan, BAV or Tuner patients, either multiple imaging modalities and measurement techniques were used or information on diameter assessment are lacking [6, 23, 37-39].

Burman et al. found similar to our study significantly larger $\mathrm{CuCu}$ than $\mathrm{CuCo}$ diameters [25]. They suggested to privilege $\mathrm{CuCo}$ diameters as they correlated best to age and body surface area and were found to correspond closely to reference TTE root measurements recorded in the Framingham Heart Study cohort. However, based on our observations, this method is associated with a considerable risk of underestimation of the largest aortic root diameter. We show that $\mathrm{CMR} \mathrm{CuCu}$ diameters are systematically larger than TTE and up to $3 \mathrm{~mm}$ larger than $\mathrm{CuCo}$ diameters. Both observations are in line with previous studies on healthy individuals, BAV and CHD patients $[21,25,26]$. Particularly in BAV patients, TTE is at risk to underestimate the maximum diameter due to an increased aortic root asymmetry. The asymmetry was significantly more important in BAV patients than in non-BAV patients and associated to a larger discrepancy of $\mathrm{CuCu}$ diameters to TTE diameters (5.5 vs. $3.3 \mathrm{~mm}$ ), similar to previous findings $[21,26]$. In contrast to TTE, $\mathrm{CT}$ and CMR show a very good agreement when determining I-I aortic root diameters as performed in our study $[21,29,38]$.

There is therefore a clear need for harmonization and a prospective validation of imaging and measurement protocols as well as thresholds for surgical intervention.

Based on our findings, we have adopted the strategy to measure aortic root diameters on the 3D self nav whole heart images which are routinely acquired during our CHD CMR scan protocol. This strategy reduces the scan time by about $10 \mathrm{~min}$ as no acquisition of a second LVOT and the cross-sectional cines of the aortic root is required $[14,20]$. In addition, as the $3 \mathrm{D}$ self nav sequence allows the evaluation of the whole cardiac and thoracic anatomy 
and vessels it can replace the anatomic localizers and the $3 \mathrm{D}$ angiography [15].

\section{Limitations}

As it is a retrospective study, the choice of slice thickness of the 2D cines was to the discretion of the physician in charge, the reason why it was not uniform and varied between 4.5 to $7 \mathrm{~mm}$, but without influence on precision $(p>0.05)$. The heterogeneity of the patient population could be criticized, however, showing also the robustness of both techniques in different pathologies and conditions and, e.g. demonstrating that aortic root evaluation in BAV patients is prone to underestimation of the correct maximum diameter when using $\mathrm{CuCo}$ diameters. Decision on the contrast media administration was to the discretion of the physician in charge of the exam. In our institution, a contrast-enhanced 3D CMR angiography is performed for all patient who undergo, in particular if for the first time, a CMR exam for assessment of the great vessels. This explains why contrast media was administered for all patients, which, however, would not be required for the sole assessment of aortic root diameters. The 3D self nav technique may also perform well without contrast as previously shown [15].

\section{Conclusions}

Both, the 2D cine and the 3D self nav technique are reliable methods for determination of aortic root diameters in $\mathrm{CHD}$ patients. Considering the higher spatial resolution and a more convenient acquisition for the patients, the $3 \mathrm{D}$ self nav technique could be privileged for the followup of patients with aortic root enlargement. In addition, $\mathrm{CuCu}$ diameters should be determined as, particularly in asymmetric aortic roots like in BAV patients, $\mathrm{CuCo}$ diameters present a considerable risk to underestimate the maximum diameter.

\section{Abbreviations \\ 3D self nav: 3D self-navigated free-breathing high-resolution whole heart sequence; BAV: Bicuspid aortic valve; bSSFP: Balanced steady-state free preces- sion; CCT: Cardiac computed tomography; CHD: Congenital heart disease; CMR: Cardiovascular magnetic resonance; CuCo: Cusp to commissure; CuCu: Cusp to cusp; ECG: Electrocardiogram; GRE: Gradient echo; HR: Heart rate; L-L: Leading to leading; LV: Left ventricle/left ventricular; LVOT: Left ventricu- lar outflow tract; MPR: Multiplanar reconstruction; O-O: Outer to outer; TTE: Transthoracic echocardiography; SD: Standard deviation.}

\section{Supplementary Information}

The online version contains supplementary material available at https://doi. org/10.1186/s12968-021-00744-1.

Additional file 1. Additional figures and tables.

\section{Acknowledgements}

Not applicable

\section{Authors' contributions}

$\mathrm{CN}$ and TR developed the study protocol, collected and analyzed the data and drafted the manuscript. DP and MS developed the 3D self nav sequence. JS was involved in the development of the study design. All authors read and approved the final manuscript.

\section{Funding}

No specific funding was obtained for this study.

\section{Availability of data and materials}

The datasets used and/or analyzed during the current study are available from the corresponding author on reasonable request.

\section{Declarations}

\section{Ethics approval and consent to participate}

The "Commission Cantonale d'Éthique de la Recherche sur l'Être Humain, Vaud" approved the project and need for consent form was waived.

\section{Consent for publication}

Not applicable.

\section{Competing interests}

$\mathrm{CN}, \mathrm{JB}, \mathrm{TR}, \mathrm{AP}, \mathrm{MS}$ and PM do not declare competing interest. DP is an employee of Siemens Healthcare and possesses stocks of Siemens Healthcare (conflict not relevant for this paper). JS receives annual funding of Bayer Healthcare, Switzerland. Dr. Tim Leiner served as a JCMR Guest Editor for this manuscript.

\section{Author details}

${ }^{1}$ Service of Cardiology, Centre de Resonance Magnétique Cardiaque, Lausanne University Hospital and University of Lausanne, Lausanne, Switzerland.

${ }^{2}$ Service of Cardiology, Adult Congenital Heart Disease Unit, Lausanne University Hospital and University of Lausanne, Lausanne, Switzerland. ${ }^{3}$ Department of Radiology, University Hospital and University of Lausanne, Lausanne, Switzerland. ${ }^{4}$ Advanced Clinical Imaging Technology, Siemens Healthcare AG, Lausanne, Switzerland.

Received: 4 December 2020 Accepted: 17 March 2021

Published online: 27 May 2021

References

1. Verma S, Siu SC. Aortic dilatation in patients with bicuspid aortic valve. N Engl J Med. 2014;370:1920-9.

2. Detaint $D$, Faivre $L$, Collod-Beroud $G$, Child AH, Loeys BL, Binquet $C$, Gautier E, Arbustini E, Mayer K, Arslan-Kirchner M, Stheneur C, Halliday D, Beroud C, Bonithon-Kopp C, Claustres M, Plauchu H, Robinson PN, Kiotsekoglou A, De Backer J, Ades L, Francke U, De Paepe A, Boileau C, Jondeau G. Cardiovascular manifestations in men and women carrying a FBN1 mutation. Eur Heart J. 2010:31:2223-9.

3. Mortensen $\mathrm{KH}$, Hjerrild BE, Stochholm K, Andersen NH, Sorensen KE, Lundorf E, Horlyck A, Pedersen EM, Christiansen JS, Gravholt CH. Dilation of the ascending aorta in Turner syndrome-a prospective cardiovascular magnetic resonance study. J Cardiovasc Magn Reson. 2011;13:24.

4. Yetman AT, Graham T. The dilated aorta in patients with congenital cardiac defects. J Am Coll Cardiol. 2009:53:461-7.

5. Erbel R, Aboyans V, Boileau C, Bossone E, Bartolomeo RD, Eggebrecht H, Evangelista A, Falk V, Frank H, Gaemperli O, Grabenwoger M, Haverich A, lung B, Manolis AJ, Meijboom F, Nienaber CA, Roffi M, Rousseau H, Sechtem U, Sirnes PA, Allmen RS, Vrints CJ and Guidelines ESCCFP. 2014 ESC Guidelines on the diagnosis and treatment of aortic diseases: Document covering acute and chronic aortic diseases of the thoracic and abdominal aorta of the adult. The Task Force for the Diagnosis and Treatment of Aortic Diseases of the European Society of Cardiology (ESC). Eur Heart J. 2014;35:2873-926. 
6. Jondeau G, Detaint D, Tubach F, Arnoult F, Milleron O, Raoux F, Delorme G, Mimoun L, Krapf L, Hamroun D, Beroud C, Roy C, Vahanian A, Boileau C. Aortic event rate in the Marfan population: a cohort study. Circulation. 2012;125:226-32.

7. Tzemos N, Therrien J, Yip J, Thanassoulis G, Tremblay S, Jamorski MT, Webb GD, Siu SC. Outcomes in adults with bicuspid aortic valves. JAMA. 2008;300:1317-25.

8. Sherrah AG, Andvik S, van der Linde D, Davies L, Bannon PG, Padang R, Vallely MP, Wilson MK, Keech AC, Jeremy RW. Nonsyndromic thoracic aortic aneurysm and dissection: outcomes with Marfan syndrome versus bicuspid aortic valve aneurysm. J Am Coll Cardiol. 2016;67:618-26.

9. Edelman RR, Chien D, Kim D. Fast selective black blood MR imaging Radiology. 1991;181:655-60.

10. Potthast S, Mitsumori L, Stanescu LA, Richardson ML, Branch K, Dubinsky TJ, Maki JH. Measuring aortic diameter with different MR techniques: comparison of three-dimensional (3D) navigated steady-state free-precession (SSFP), 3D contrast-enhanced magnetic resonance angiography (CE-MRA), 2D T2 black blood, and 2D cine SSFP. J Magn Reson Imaging. 2010;31:177-84.

11. Lang RM, Badano LP, Mor-Avi V, Afilalo J, Armstrong A, Ernande L, Flachskampf FA, Foster E, Goldstein SA, Kuznetsova T, Lancellotti P, Muraru D, Picard MH, Rietzschel ER, Rudski L, Spencer KT, Tsang W, Voigt JU. Recommendations for cardiac chamber quantification by echocardiography in adults: an update from the American Society of Echocardiography and the European Association of Cardiovascular Imaging. J Am Soc Echocardiogr. 2015;28(1-39):e14.

12. Plonek T, Berezowski M, Kurcz J, Podgorski P, Sasiadek M, Rylski B, Mysiak A, Jasinski M. The evaluation of the aortic annulus displacement during cardiac cycle using magnetic resonance imaging. BMC Cardiovasc Disord. 2018;18:154.

13. Goldstein SA, Evangelista A, Abbara S, Arai A, Asch FM, Badano LP, Bolen MA, Connolly HM, Cuellar-Calabria H, Czerny M, Devereux RB, Erbel RA, Fattori R, Isselbacher EM, Lindsay JM, McCulloch M, Michelena HI, Nienaber CA, Oh JK, Pepi M, Taylor AJ, Weinsaft JW, Zamorano JL, Dietz H, Eagle K, Elefteriades J, Jondeau G, Rousseau H, Schepens M. Multimodality imaging of diseases of the thoracic aorta in adults: from the American Society of Echocardiography and the European Association of Cardiovascular Imaging: endorsed by the Society of Cardiovascular Computed Tomography and Society for Cardiovascular Magnetic Resonance. J Am Soc Echocardiogr. 2015;28:119-82.

14. Rutz T, Wustmann K, Prsa M, Vallée JP, Donner B, Bremerich J, Deluigi C, Zellweger M, Tobler D, Kellenberger C, Wahl A, Monney P, Buser P, Gudinchet F, Schwitter J, Valsangiacomo BE. Cardiac magnetic resonance imaging in congenital heart disease. Cardiovasc Med. 2016;19:176-84.

15. Monney P, Piccini D, Rutz T, Vincenti G, Coppo S, Koestner SC, Sekarski N, Di Bernardo S, Bouchardy J, Stuber M, Schwitter J. Single centre experience of the application of self navigated 3D whole heart cardiovascular magnetic resonance for the assessment of cardiac anatomy in congenital heart disease. J Cardiovasc Magn Reson. 2015;17:55.

16. Piccini D, Littmann A, Nielles-Vallespin S, Zenge MO. Respiratory selfnavigation for whole-heart bright-blood coronary MRI: methods for robust isolation and automatic segmentation of the blood pool. Magn Reson Med. 2012;68:571-9.

17. Piccini D, Monney P, Sierro C, Coppo S, Bonanno G, van Heeswijk RB, Chaptinel J, Vincenti G, de Blois J, Koestner SC, Rutz T, Littmann A, Zenge MO, Schwitter J, Stuber M. Respiratory self-navigated postcontrast whole-heart coronary MR angiography: initial experience in patients. Radiology. 2014;270:378-86.

18. Piccini D, Littmann A, Nielles-Vallespin S, Zenge MO. Spiral phyllotaxis: the natural way to construct a 3D radial trajectory in MRI. Magn Reson Med. 2011;66:1049-56.

19. Stehning C, Bornert P, Nehrke K, Eggers H, Stuber M. Free-breathing whole-heart coronary MRA with 3D radial SSFP and self-navigated image reconstruction. Magn Reson Med. 2005;54:476-80.

20. Fratz S, Chung T, Greil GF, Samyn MM, Taylor AM, Valsangiacomo Buechel ER, Yoo SJ, Powell AJ. Guidelines and protocols for cardiovascular magnetic resonance in children and adults with congenital heart disease: SCMR expert consensus group on congenital heart disease. J Cardiovasc Magn Reson. 2013;15:51.
21. Torres FS, Windram JD, Bradley TJ, Wintersperger BJ, Menezes R, Crean AM, Colman JM, Silversides CK, Wald RM. Impact of asymmetry on measurements of the aortic root using cardiovascular magnetic resonance imaging in patients with a bicuspid aortic valve. Int J Cardiovasc Imaging. 2013;29:1769-77.

22. Bland JM, Altman DG. Statistical methods for assessing agreement between two methods of clinical measurement. Lancet. 1986;1:307-10

23. Michelena HI, Khanna AD, Mahoney D, Margaryan E, Topilsky Y, Suri RM, Eidem B, Edwards WD, Sundt TM 3rd, Enriquez-Sarano M. Incidence of aortic complications in patients with bicuspid aortic valves. JAMA. 2011;306:1104-12.

24. Veldhoen S, Behzadi C, Lenz A, Henes FO, Rybczynski M, von Kodolitsch Y, Bley TA, Adam G, Bannas P. Non-contrast MR angiography at 1.5 Tesla for aortic monitoring in Marfan patients after aortic root surgery. J Cardiovasc Magn Reson. 2017;19:82.

25. Burman ED, Keegan J, Kilner PJ. Aortic root measurement by cardiovascular magnetic resonance: specification of planes and lines of measurement and corresponding normal values. Circ Cardiovasc Imaging. 2008;1:104-13.

26. Nejatian A, Yu J, Geva T, White MT, Prakash A. Aortic measurements in patients with aortopathy are larger and more reproducible by cardiac magnetic resonance compared with echocardiography. Pediatr Cardiol. 2015;36:1761-73.

27. Rodriguez-Palomares JF, Teixido-Tura G, Galuppo V, Cuellar H, Laynez A, Gutierrez L, Gonzalez-Alujas MT, Garcia-Dorado D, Evangelista A. Multimodality assessment of ascending aortic diameters: comparison of different measurement methods. J Am Soc Echocardiogr. 2016;29(819-826):e4

28. Krishnam MS, Tomasian A, Malik S, Desphande V, Laub G, Ruehm SG. Image quality and diagnostic accuracy of unenhanced SSFP MR angiography compared with conventional contrast-enhanced MR angiography for the assessment of thoracic aortic diseases. Eur Radiol. 2010;20:1311-20.

29. Weinrich JM, Avanesov M, Lenz A, Tahir E, Henes FO, Schoennagel BP, Rybczinsky M, Adam G, von Kodolitsch Y, Bannas P. Reliability of noncontrast magnetic resonance angiography-derived aortic diameters in Marfan patients: comparison of inner vs. outer vessel wall measurements. Int J Cardiovasc Imaging. 2020;8:97.

30. von Knobelsdorff-Brenkenhoff F, Gruettner H, Trauzeddel RF, Greiser A, Schulz-Menger J. Comparison of native high-resolution 3D and contrast-enhanced MR angiography for assessing the thoracic aorta. Eur Heart J Cardiovasc Imaging. 2014;15:651-8.

31. Bhatla P, Nielsen JC. Cardiovascular magnetic resonance as an alternate method for serial evaluation of proximal aorta: comparison with echocardiography. Echocardiography. 2013;30:713-8.

32. Ginami G, Bonanno G, Schwitter J, Stuber M, Piccini D. An iterative approach to respiratory self-navigated whole-heart coronary MRA significantly improves image quality in a preliminary patient study. Magn Reson Med. 2016;75:1594-604.

33. Heerfordt J, Stuber M, Maillot A, Bianchi V, Piccini D. A quantitative comparison between a navigated Cartesian and a self-navigated radial protocol from clinical studies for free-breathing 3D whole-heart bSSFP coronary MRA. Magn Reson Med. 2020:84:157-69.

34. Rutz T, Piccini D, Coppo S, Chaptinel J, Ginami G, Vincenti G, Stuber M, Schwitter J. Improved border sharpness of post-infarct scar by a novel self-navigated free-breathing high-resolution 3D whole-heart inversion recovery magnetic resonance approach. Int J Cardiovasc Imaging. 2016;32:1735-44.

35. Hiratzka LF, Bakris GL, Beckman JA, Bersin RM, Carr VF, Casey DE, Eagle KA, Hermann LK, Isselbacher EM, Kazerooni EA, Kouchoukos NT, Lytle BW, Milewicz DM, Reich DL, Sen S, Shinn JA, Svensson LG, Williams DM, American College of Cardiology Foundation/American Heart Association Task Force on Practice G, American Association for Thoracic $S$, American College of R, American Stroke A, Society of Cardiovascular A, Society for Cardiovascular A, Interventions, Society of Interventional R, Society of Thoracic S and Society for Vascular M. 2010 ACCF/AHA/ AATS/ACR/ASA/SCA/SCAI/SIR/STS/SVM guidelines for the diagnosis and management of patients with Thoracic Aortic Disease: a report of the American College of Cardiology Foundation/American Heart Association Task Force on Practice Guidelines, American Association for Thoracic Surgery, American College of Radiology, American Stroke 
Association, Society of Cardiovascular Anesthesiologists, Society for Cardiovascular Angiography and Interventions, Society of Interventional Radiology, Society of Thoracic Surgeons, and Society for Vascular Medicine. Circulation. 2010;121:e266-369.

36. Petersen SE, Khanji MY, Plein S, Lancellotti P, Bucciarelli-Ducci C. European Association of Cardiovascular Imaging expert consensus paper: a comprehensive review of cardiovascular magnetic resonance normal values of cardiac chamber size and aortic root in adults and recommendations for grading severity. Eur Heart J Cardiovasc Imaging. 2019;20:1321-31.

37. Carlson M, Airhart N, Lopez L, Silberbach M. Moderate aortic enlargement and bicuspid aortic valve are associated with aortic dissection in Turner syndrome: report of the international turner syndrome aortic dissection registry. Circulation. 2012;126:2220-6.

38. Frazao C, Tavoosi A, Wintersperger BJ, Nguyen ET, Wald RM, Ouzounian M, Hanneman K. Multimodality assessment of thoracic aortic dimensions: comparison of computed tomography angiography, magnetic resonance imaging, and echocardiography measurements. J Thorac Imaging. 2020;2:44.

39. Weinsaft JW, Devereux RB, Preiss LR, Feher A, Roman MJ, Basson CT, Geevarghese A, Ravekes W, Dietz HC, Holmes K, Habashi J, Pyeritz RE, Bavaria J, Milewski K, LeMaire SA, Morris S, Milewicz DM, Prakash S, Maslen C, Song HK, Silberbach GM, Shohet RV, McDonnell N, Hendershot T, Eagle KA, Asch FM, Investigators GR. Aortic dissection in patients with genetically mediated aneurysms: incidence and predictors in the GenTAC registry. J Am Coll Cardiol. 2016;67:2744-54.

\section{Publisher's Note}

Springer Nature remains neutral with regard to jurisdictional claims in published maps and institutional affiliations.
Ready to submit your research? Choose BMC and benefit from:

- fast, convenient online submission

- thorough peer review by experienced researchers in your field

- rapid publication on acceptance

- support for research data, including large and complex data types

- gold Open Access which fosters wider collaboration and increased citations

- maximum visibility for your research: over $100 \mathrm{M}$ website views per year

At BMC, research is always in progress.

Learn more biomedcentral.com/submissions 